\title{
Uma revisão da distribuição de Ocotea curucutuensis J.B. Baitello na região sudeste do Brasil
}

\author{
Frederico Alexandre Roccia Dal Pozzo Arzolla ${ }^{1,2,4}$, João Batista Baitello ${ }^{1}$, \\ George John Shepherd ${ }^{3}$ Gláucia Cortez Ramos de Paula ${ }^{1} \&$ Ricardo Bertoncello ${ }^{2}$ \\ ${ }^{1}$ Instituto Florestal, SMA-SP, Rua do Horto 931, CEP 02377-000, São Paulo, SP, Brasil \\ ${ }^{2}$ Programa de Pós-Graduação em Biologia Vegetal, Universidade Estadual de Campinas - UNICAMP, \\ CP 6109, CEP 13083-970, Campinas, SP, Brasil \\ ${ }^{3}$ Departamento de Botânica, Instituto de Biologia, Universidade Estadual de Campinas - UNICAMP, \\ CP 6109, CEP 13083-970, Campinas, SP, Brasil \\ ${ }^{4}$ Autor para correspondência: Frederico Alexandre Roccia Dal Pozzo Arzolla, \\ e-mail:fredericoarzolla@uol.com.br
}

ARZOLLA, F.A.R.D.P., BAITELLO, J.B., SHEPHERD, G.J., PAULA, G.C.R. \& BERTONCELLO, R. 2009. A revised distribution for Ocotea curucutuensis J.B. Baitello in southeastern Brazil. Biota Neotrop., 9(1): http://www.biotaneotropica.org.br/v9n1/en/abstract?article+bn00209012009.

\begin{abstract}
This analysis presents a revised distribution for Ocotea curucutuensis J.B. Baitello in southeastern Brazil. Because of its strong similarity with Ocotea spixiana (Nees) Mez this species remained unrecognized for over a hundred years after the first collection by A. F. M. Glaziou in 1888, but it was recently shown to be a distinct species. Although the species was originally described from material from the Serra do Mar in São Paulo, subsequent new collections and a wider investigation of herbarium material have shown that it is, in fact, quite widespread, especially in the Serra do Mar and Serra da Mantiqueira and that it is necessary to revise and enlarge the known distribution. O. curucutuensis has a wide distribution in forests in the higher parts of the Serra do Mar and Serra da Mantiqueira, in the states of Rio de Janeiro and São Paulo, in the southeast of Brazil, while $O$. spixiana has a more northerly distribution.
\end{abstract}

Keywords: cloud forest, high-altitude forest, Serra do Mar, Serra da Mantiqueira, Ocotea curucutuensis.

ARZOLLA, F.A.R.D.P., BAITELLO, J.B., SHEPHERD, G.J., PAULA, G.C.R. \& BERTONCELLO, R. 2009. Uma revisão da distribuição de Ocotea curucutuensis J.B. Baitello na região sudeste do Brasil. Biota Neotrop., 9(1): http://www.biotaneotropica.org.br/v9n1/pt/abstract?article+bn00209012009.

Resumo: Este trabalho apresenta uma revisão da distribuição de Ocotea curucutuensis J.B. Baitello, na Região Sudeste do Brasil, ampliando substancialmente a extensão conhecida da sua ocorrência. Pela semelhança com Ocotea spixiana (Nees) Mez, essa espécie permaneceu confundida com esta e desconhecida por mais de cem anos, desde a primeira coleta por A. F. M. Glaziou, em 1888. Com base em novos registros para a Serra do Mar e para a Serra da Mantiqueira, sua distribuição foi revisada e ampliada. O. curucutuensis apresenta uma ampla distribuição nas florestas das partes altas da Serra do Mar e da Serra da Mantiqueira, nos Estados de São Paulo e Rio de Janeiro, no sudeste do Brasil, enquanto O. spixiana possui uma distribuição mais ao norte do País.

Palavras-chave: matas nebulares, florestas de altitude, Serra do Mar, Serra da Mantiqueira, Ocotea curucutuensis. 


\section{Introdução}

A família Lauraceae é pantropical, com poucos membros nas regiões temperadas. O número exato de espécies é desconhecido, mas estima-se que está entre 2.500 e 3 mil, distribuídos em 50 gêneros (Rohwer, 1993). No Brasil são 21 gêneros e cerca de 360 espécies, mas este número pode estar subestimado. No Estado do Rio de Janeiro, Quinet (2005) menciona a ocorrência de 109 espécies e 16 gêneros. No Estado de São Paulo, Baitello (2003) relata 91 espécies e 13 gêneros. Ocotea Aubl. é um dos gêneros de maior riqueza de espécies nas florestas do Estado, com 45 espécies (Baitello, 2003).

Ocotea curucutuensis J.B. Baitello foi descrita com base em materiais da Serra do Mar, no extremo sul do município de São Paulo, na divisa com Itanhaém (Baitello, 2001). Essa espécie tem como características de destaque folhas lanceoladas, coriáceas, com densa pilosidade ferrugínea na face abaxial, especialmente nas folhas jovens. A nervura central da folha apresenta-se proeminente e espessada no terço inferior do limbo. O pecíolo também é espessado e achatado (Figura 1b).

Assemelha-se à $O$. spixiana (Nees) Mez pelas dimensões e estrutura de suas flores e pela pilosidade ferrugínea da face abaxial de suas folhas (Baitello, 2001). Ambas são unissexuadas. Por essa semelhança, Ocotea curucutuensis permaneceu confundida com aquela e desconhecida por mais de cem anos, desde que o primeiro material botânico foi coletado pelo naturalista francês A. F. M. Glaziou, em
1880, na Serra dos Órgãos, Estado do Rio de Janeiro, que se encontra depositado no Herbário do Instituto de Pesquisas Jardim Botânico do Rio de Janeiro (RB).

Ocotea curucutuensis difere de $O$. spixiana especialmente pela densa pilosidade fortemente ferrugíneo-avermelhada na face abaxial da folha, em geral cobrindo toda a epiderme; estames mais longos e frutos de duas a quatro vezes maior e densamente pilosos em toda a sua extensão (Baitello, 2003) (Figuras 1a e 1b).

Somente mais recentemente, após a coleta de materiais na Serra da Mantiqueira, em Pindamonhangaba e Campos do Jordão, e na Serra do Mar, em Ubatuba, pôde ser inferida a nova distribuição da espécie e, a partir daí, foi realizada uma busca de possíveis coletas depositadas em herbários regionais.

\section{Materiais e Métodos}

Para a análise sobre a distribuição e o habitat das espécies, foram levantados os registros de Ocotea curucutuensis e Ocotea spixiana, na base de dados do Species-link do Centro de Referências em Informação Ambiental (CRIA). Com base na lista dos registros para as duas espécies, foram examinadas as exsicatas depositadas em três herbários representativos da Região Sudeste: Instituto Florestal (SPSF), Instituto de Pesquisas Jardim Botânico do Rio de Janeiro (RB) e Universidade Estadual de Campinas (UEC).
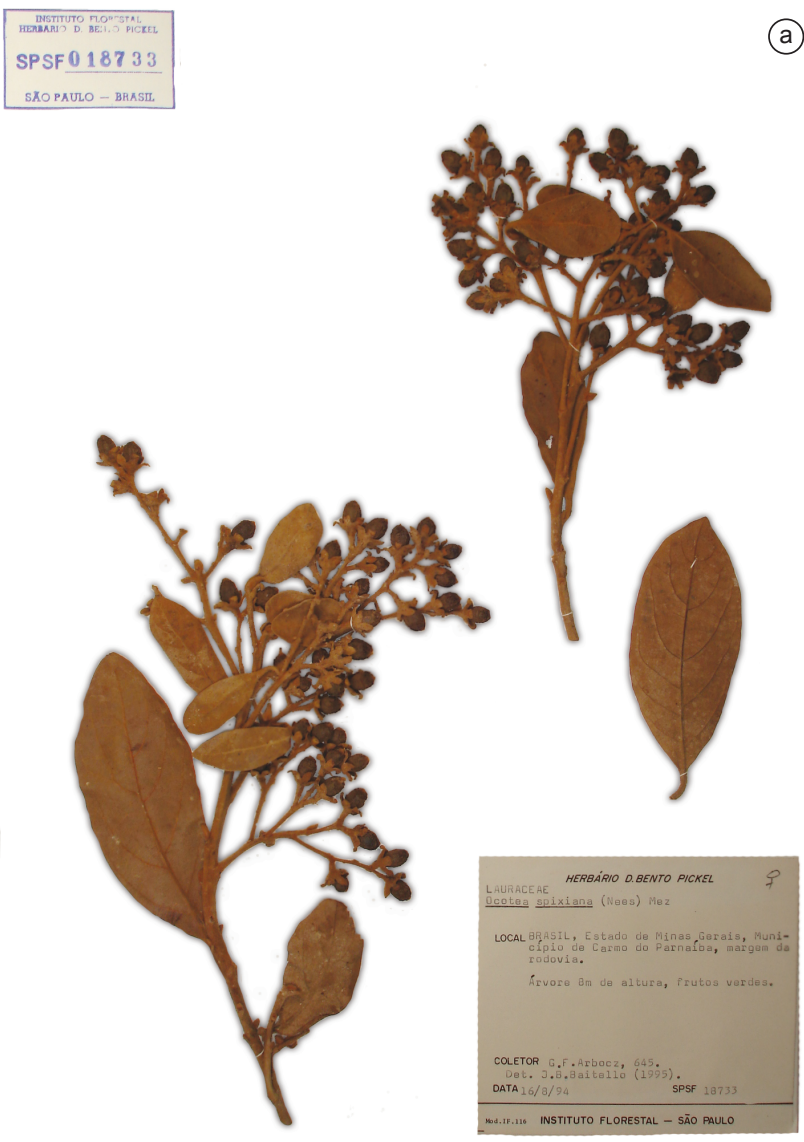

(a)

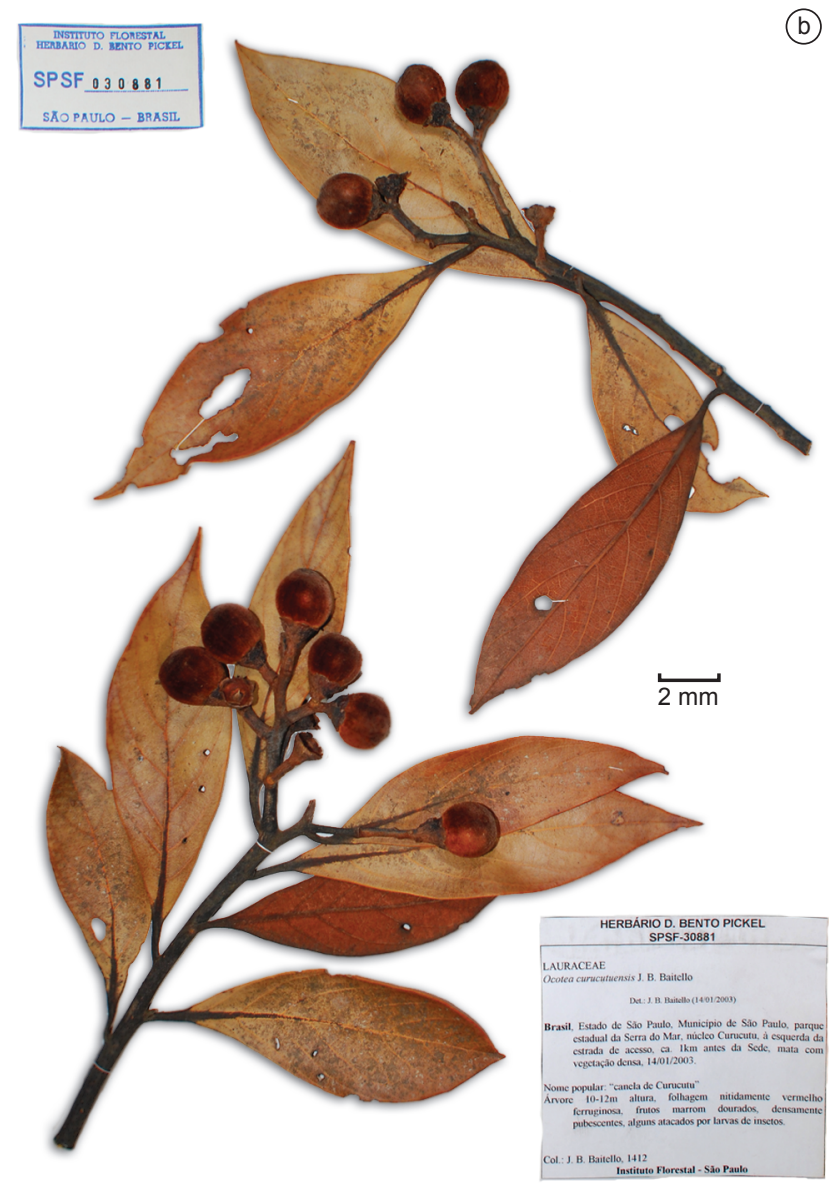

Figura 1. Exsicatas de a) Ocotea spixiana; e b) Ocotea curucutuensis: nota-se a marcante diferença no tamanho dos frutos adultos (Foto: J.B.Baitello).

Figure 1. Specimens of a) Ocotea spixiana; and b) Ocotea curucutuensis: note the marked difference in the size of the mature fruit (Photograph: J.B. Baitello). 
Para o georeferenciamento dos registros botânicos de O. curucutuensis, foram levantados pontos em cartas planialtimétricas, a partir das localidades mencionadas nas fichas de herbário, ou em campo com um aparelho de GPS (Global Positioning System). Para $O$. spixiana, foi utilizada a coordenada da sede do município, disponível na base de dados do Species-link do CRIA.

\section{Resultados}

Apresentam-se três novos registros para a espécie em São Paulo: nas partes altas da Serra da Mantiqueira, em Pindamonhangaba e Campos do Jordão, e da Serra do Mar, em Ubatuba (Figuras 2 e 3). A partir desses registros, levantou-se a hipótese de que a distribuição da espécie poderia ser mais ampla, e que os registros de $O$. spixiana para a Serra do Mar e para a Serra da Mantiqueira, no Estado do Rio de Janeiro, provavelmente fossem de O. curucutuensis. A hipótese aventada foi corroborada após a análise dos materiais provenientes desses registros, especialmente as consultas ao Herbário RB.

Depois da revisão dos materiais, constatou-se que Ocotea curucutuensis apresenta distribuição em vários pontos das Serras do Mar e da Mantiqueira, a seguir: SÃO PAULO - Campos do Jordão, III.2007, F.A.R.D.P. Arzolla \& G.C.R. Paula n ${ }^{\circ} 1.073$ (SPSF); Campos do Jordão, I.2008, F.A.R.D.P. Arzolla no 1.248
(SPSF); Campos do Jordão, I.2008, J.B. Baitello nº 2.004 (SPSF); Cubatão, V.2001, J.B. Baitello no 989 (SPSF); Cubatão, V.2001, J.B. Baitello n 990; Itanhaém, III.1999, P. Affonso nº 366 (PMSP, SPSF); Pindamonhangaba, IX.2005, F.A.R.D.P. Arzolla \& J.D. Braz $\mathrm{n}^{\circ} 1.018$ (SPSF); São Paulo, I.1995, M. Alonso et al. nº 64 (SPSF); São Paulo, III. 1998, P. Affonso n 168 (PMSP, SPSF); São Paulo, I.2001, J.B. Baitello n 978 (SPSF); São Paulo, IV.2001, J.B. Baitello n 991 (SPSF); São Paulo, I.2003, J.B. Baitello nº 1412 (SPSF); São Paulo, XII.2003, J.B. Baitello n ${ }^{0} 1.641$ (SPSF); Ubatuba, IV.2007, R. Bertoncello n 282 (UEC); Ubatuba, V.2007, R. Bertoncello s.n ${ }^{\circ}$ (UEC); Ubatuba, V.2007, R. Bertoncello no 350 (UEC); Ubatuba, VI.2007, R. Bertoncello n ${ }^{\circ} 482$ (UEC); Ubatuba, IV.2007, R. Bertoncello ${ }^{\circ} 516$ (UEC); RIO DE JANEIRO - Serra dos Órgãos, III.1888, A.F.M. Glaziou n 17.740 (RB); Nova Friburgo, XI.1986, G. Martinelli et al. no 11.931 (RB); Paraty, XI.1990, C. Farney et al. $\mathrm{n}^{\circ} 2.584$ (RB); Paraty, IV.1994, R. Marquete $\mathrm{n}^{\circ} 1.640$ (RB); Parque Nacional de Itatiaia, W.D. Barros no 909 (RB).

Foram selecionados os seguintes registros de Ocotea spixiana: BAHIA - Caitité, IV.1980, R.M. Harley no 21.337 (SPSF); Rio de Contas, IV.1999, F. Nascimento n ${ }^{\circ} 196$ (HST); DISTRITO FEDERAL - Brasília, III.1984, B.A.S. Pereira no 941 (RB); Brasília, IV.1962, E.P. Heringer n ${ }^{\circ} 8.913$ (RB); Brasília, III.1998, A.C. Sevilha n ${ }^{\circ} 1.760$
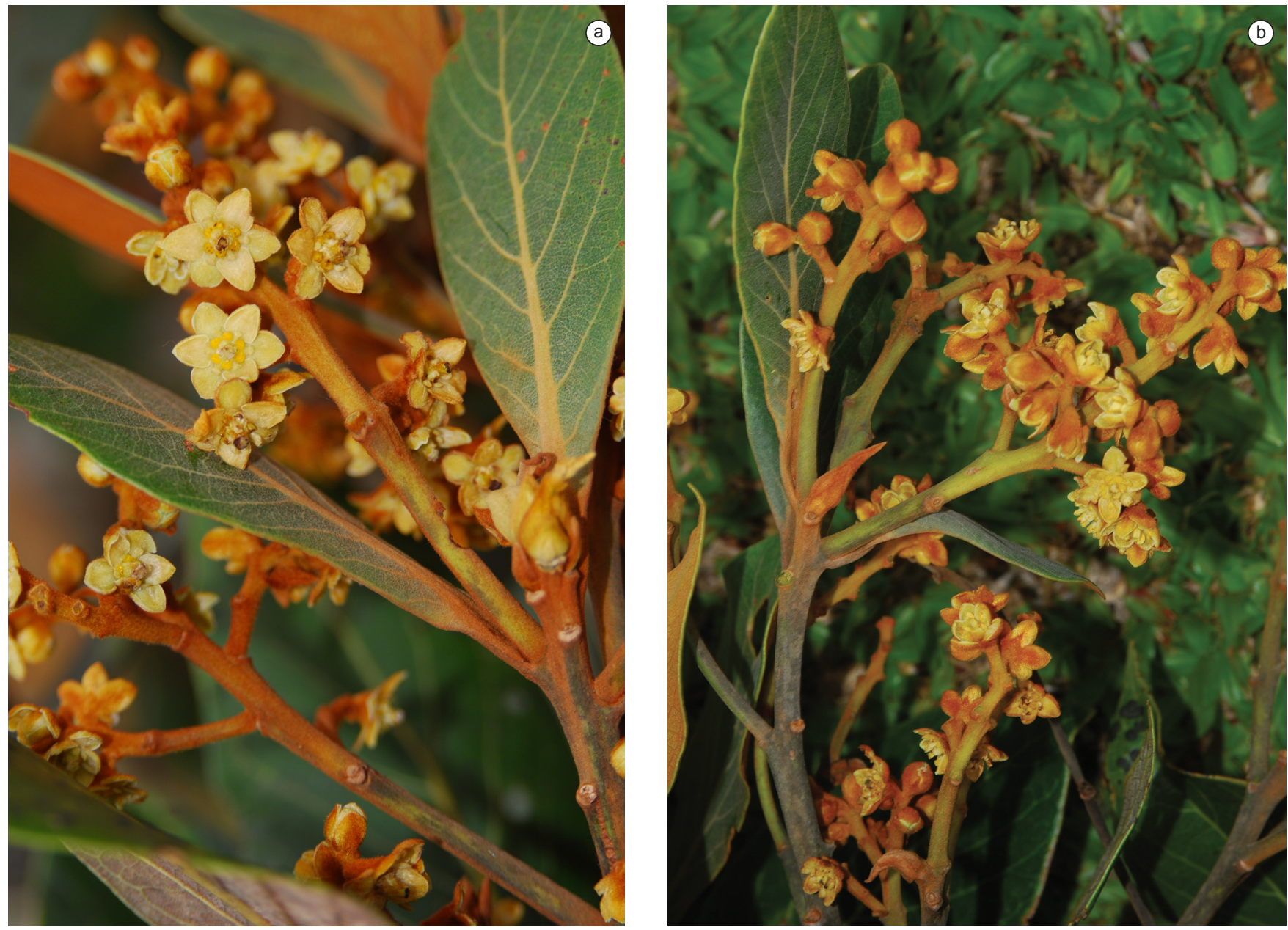

Figura 2. Detalhe das inflorescências de um indivíduo a) feminino e b) masculino de Ocotea curucutuensis, na região do Pico do Itapeva, Campos do Jordão, SP. (Foto: J.B. Baitello).

Figure 2. Detail of the inflorescences of a) female and b) male individuals of Ocotea curucutuensis near the Pico de Itapeva, Campos do Jordão, São Paulo (Photograph: J.B. Baitello). 


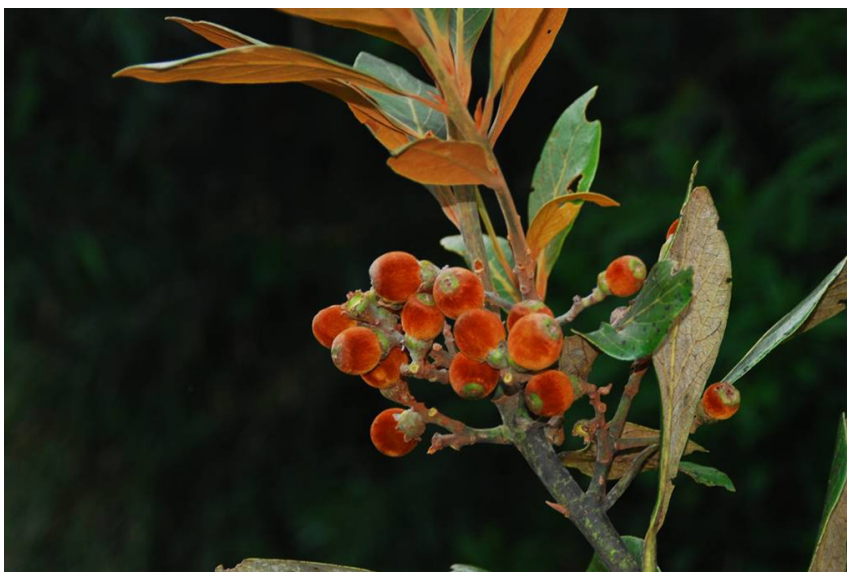

Figura 3. Detalhe de infrutescência de Ocotea curucutuensis, na região do Pico do Itapeva, Campos do Jordão, SP. (Foto: J.B.Baitello).

Figure 3. Detail of fruits of Ocotea curucutuensis near the Pico do Itapeva, Campos do Jordão, São Paulo. (Photograph: J.B. Baitello).

(IBGE); Brasília, VIII.1995, M.A. Silva n 2.710 (IBGE); Brasília, V.1980, E.P. Heringer et al no 4.881 (IBGE); ESPÍRITO SANTO Santa Teresa, VI.1999, L. Kollmann et al. n 2.633 (MBML); GOIÁS - Alto Paraíso, VI.1965, F.R. Rosa nº 76 (RB); Caldas Novas, IV.1993, S.P. Cordovil et al. no 283 (SPSF); Goiânia, IV.2007, R. Tsuji et al. $\mathrm{n}^{\circ} 1.929$ (SPSF); Mineiros, IX.1993, F. Bucci no 59 (INPA); Niquelândia, VIII.1995, M.L. Fonseca et al. n 440; Pirenópolis, VI.2000, D. Willberg no 141 (MBM); São João d'Aliança, V.1975, G. Hatschbach $\mathrm{n}^{\circ} 36.724$ (MBM); MATO GROSSO DO SUL - Naviraí, VIII.1980, P.P. Furtado n ${ }^{\circ} 13$ (RB); MINAS GERAIS - Araguari, V.1992, G.M. Araújo n 910 (SPSF); Carmo do Parnaíba, G.F. Arbocz nº 645, VIII.1994 (SPSF); Conselheiro da Mata, VI.1985, F.R. Martins et al. s.n (UEC); Datas, III.1987, G. Hatschbach n ${ }^{\circ} 51.081$ (MBM); Diamantina, VI.1985, F. de Barros n 1.985 (SP); Faria Lemos, II.2000, L.S. Leoni no 4.363 (RB); Grão Mogol, III.1980, G. Hatschbach $n^{\circ} 42.855$ (MBM); Itabirito, IV.1994, W.A. Teixeira s.n (SPSF); Ouro Branco, V.1988, M.T.K. Campos et al. s.n ${ }^{\circ}$ (SPSF); Ouro Preto, III.1971, D. Pedroso s.n ${ }^{\circ}$. (RB); Ouro Preto, I.1995, F.G. Lorea \& M. Gorgulho $\mathrm{n}^{\circ} 5.574$ (SPSF); Patrocínio, V.1963, M. Magalhães $\mathrm{n}^{\circ}$ 17.347 (NYBG); Rio Vermelho, VII.1985, R.M. Silva et al. nº 7.816 (RB); Santana do Riacho, X.1999, J.A. Lombardi no 3.211 (MBM); Turmalina, V.1979, V.F. Ferreira no 783 (RB); Uberlândia, X.1992, J.N. Nakajima et al. s.n ${ }^{\circ}$ (SPSF).

A distribuição de Ocotea curucutuensis conhecida até o momento abrange áreas nos Estados de São Paulo e Rio de Janeiro, na Serra do Mar, na Serra da Mantiqueira e na Serra dos Órgãos. A sua ocorrência em Minas Gerais é bastante provável (Figura 4).

Ocotea spixiana tem como distribuição principal o Distrito Federal, Goiás e Minas Gerais, ocorrendo também nos Estados da Bahia, do Espírito Santo e do Mato Grosso do Sul (Figura 4).

As fitofisionomias registradas para $O$. spixiana são principalmente o Cerrado típico, o Cerradão, os Campos Rupestres e a Floresta Estacional Semidecidual, enquanto que $O$. curucutuensis ocorre, principalmente, na Floresta Ombrófila Densa Alto-Montana.

\section{Discussão}

As áreas de ocorrência de $O$. curucutuensis encontram-se quase em sua totalidade inseridas em Unidades de Conservação - UC. No Estado de São Paulo, há registros em três localidades do Parque Estadual da Serra do Mar; nos Núcleos Curucutu, Itutinga-Pilões e

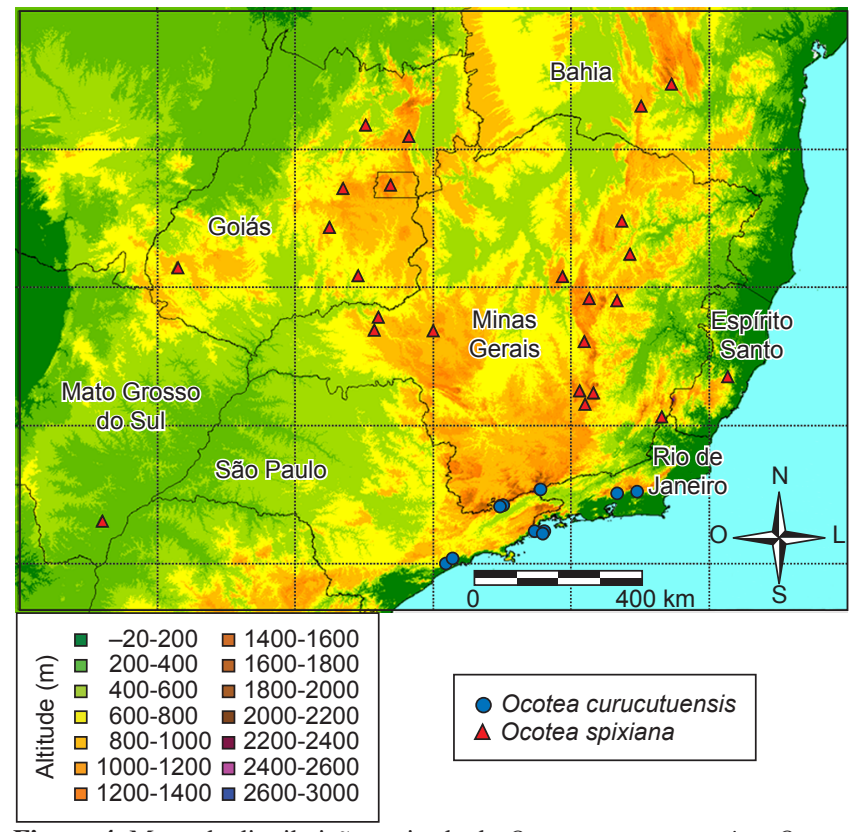

Figura 4. Mapa da distribuição revisada de Ocotea curucutuensis e Ocotea spixiana.

Figure 4. Map of Ocotea curucutuensis and Ocotea spixiana revised distribution.

Picinguaba. No Estado do Rio de Janeiro, essa espécie é encontrada nos Parques Nacionais do Itatiaia e Serra dos Órgãos, nas Reservas Ecológicas de Macaé de Cima e da Juatinga e no Parque Estadual dos Três Picos, criado em 2002, em áreas de Floresta Ombrófila Densa Montana a Alto-Montana (Rocha et al., 2003). Tais locais são em geral de difícil acesso, podendo ser alcançados quase que exclusivamente por trilhas.

Os registros fora de UC são da região do Pico do Itapeva, em Campos do Jordão, onde se encontrou uma alta abundância de indivíduos de $O$. curucutuensis, e na parte alta da Fazenda São Sebastião do Ribeirão Grande, em Pindamonhangaba, que é uma reserva da empresa Votorantim Celulose e Papel - VCP, destinada à conservação. Até o momento não há registros para o Parque Estadual de Campos do Jordão (Figura 5).

Dentre as áreas de ocorrência, a região do Pico do Itapeva é aquela que se encontra mais ameaçada. Pelo Código Florestal (Lei Federal no 4.771/1965), essa área situa-se em Área de Preservação Permanente - APP por localizar-se acima de $1.800 \mathrm{~m}$. Esse tipo de APP não tem restringido efetivamente a supressão de florestas e a conversão para outros usos na região do Pico do Itapeva. Tal área é de fácil acesso e está sob forte pressão antrópica, pela substituição de florestas nativas para a instalação de empreendimentos residenciais ou hoteleiros, e atividades agrossilvopastoris, o que indica a necessidade de adoção de medidas efetivas de proteção.

O. curucutuensis é considerada uma espécie ameaçada de extinção no Estado de São Paulo, na categoria vulnerável, conforme a Resolução SMA no 48/2004 (São Paulo, 2004), o que deve ser levado em consideração quando da análise de processos de licenciamento ambiental nessa região.

A quase totalidade da crista da Serra da Mantiqueira, entre o P.N. do Itatiaia e o P.E. de Campos do Jordão, está inserida na proposta de criação do Parque Nacional Altos da Mantiqueira (Sivelli, 2007). Caso venha a ser criada, essa UC protegerá importantes populações de Ocotea curucutuensis existentes nessa região, entre os municípios de Pindamonhangaba e Campos do Jordão. 
Os registros indicam que $O$. curucutuensis ocorre, principalmente, no interior da Floresta Ombrófila Densa Alto-Montana; a partir dos 800 m, na Serra do Mar, e a partir dos 1.600 m, na Serra da Mantiqueira. Um único registro indica a ocorrência a uma altitude de $60 \mathrm{~m}$ em Paraty, no Rio de Janeiro.

A maior parte dos registros mostra que se trata de uma espécie de porte baixo, com altura em torno dos $10 \mathrm{~m}$. Observações de campo revelam que a espécie ocupa o dossel da floresta, podendo ultrapassá-lo, como emergente. Um dos registros menciona a presença de um grande indivíduo dessa espécie, com diâmetro aproximado de $3 \mathrm{~m}$, em área de transição entre a Floresta Montana e a Alto-Montana, na vertente sul da Serra da Mantiqueira, na reserva da VCP (Figuras 5 e 6).

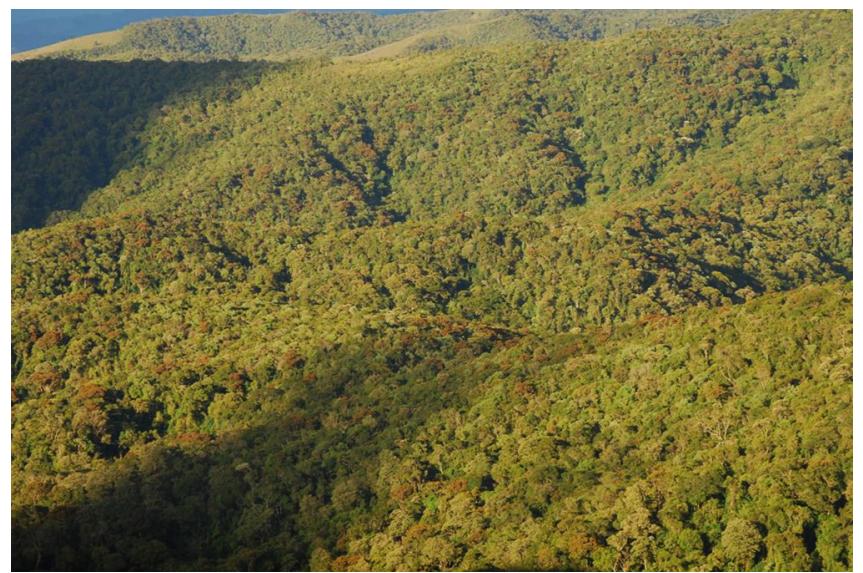

Figura 5. Aspecto da Floresta Ombrófila Densa Alto-Montana, na região do Pico do Itapeva, entre Campos de Jordão e Pindamonhangaba, São Paulo. As copas ferrugíneas identificam Ocotea curucutuensis e sua alta freqüência na área. (Foto: J.B.Baitello).

Figure 5. View of the High Montane Rain Forest near the Pico do Itapeva between Campos do Jordão and Pindamonhangaba, São Paulo. The ferrugineous-coloured tree-canopies are all Ocotea curucutuensis and show the high frequency of this species in the area. (Photograph: J.B. Baitello).

\section{Conclusões}

A distribuição de Ocotea curucutuensis foi revisada, expandindose dos planaltos do reverso da Serra do Mar, próximos à cidade de São Paulo, para outras partes altas da Serra do Mar, entre São Paulo e Paraty; da Serra dos Órgãos, e da Serra da Mantiqueira, entre Campos do Jordão e Itatiaia, nos Estados de São Paulo e Rio de Janeiro. A sua ocorrência em Minas Gerais é bastante provável.

Ocotea spixiana apresenta uma distribuição centrada no Distrito Federal, Goiás e Minas Gerais, com nítida preferência por ambientes mais secos, enquanto O. curucutuensis ocorre na Floresta Ombrófila Densa Alto-Montana, em ambientes mais úmidos.

A grande semelhança na estrutura e nas dimensões das flores dessas duas espécies pode revelar uma origem comum e uma especiação nos ambientes respectivos.

\section{Referências Bibliográficas}

BAITELLO, J.B. 2001. Novas espécies de Lauraceae para a Flora brasileira. Acta Bot. Bras. 15(3):445-450.

BAITELLO, J.B. 2003. Lauraceae. In: Wanderley, M.G.L.; Shepherd, G.J.; Melhem, T.S.; Giulietti, A.M.; Kirizawa, M. (Eds.). Flora Fanerogâmica do Estado de São Paulo. RiMa, São Carlos, v. 3, p. 149-223.

QUINET, A. 2005. Sinopse taxonômica da família Lauraceae no Estado do Rio de Janeiro, Brasil. Acta Bot. Bras. 19(3):563-572.

ROCHA, C.F.D.; BERGALLO, H.G.; ALVES, M.A.S.; VAN SLUYS, M. 2003. A biodiversidade nos grandes remanescentes florestais do Estado do Rio de Janeiro e nas restingas da Mata Atlântica. RiMa, São Carlos, $160 \mathrm{p}$.

ROHWER, J.G. 1993. Lauraceae. In: Kubitzki, K.; Rohwer, J.G. \& Bittrich, V. (Eds.). The families and genera of vascular plants. Springer-Verlag, Berlim, v. 2, p. 366-391.

SÃO PAULO (Estado). Secretaria do Meio Ambiente. 2004. Resolução SMA $\mathrm{n}^{\circ} 48$, de 21 de setembro de 2004. Disponível em: <http: Ilwww.cetesb. sp.gov.br/icenciamento/ legislaçao/ resoluções/2004_Res_SMA_48. pdf>. Acesso em: 2 Abr. 2007.

SIVELLI, F.R. 2007. Proposta de criação de uma unidade de conservação de proteção integral na Serra da Mantiqueira (São Paulo, Minas Gerais e Rio de Janeiro). IBAMA, São Paulo, 61p.

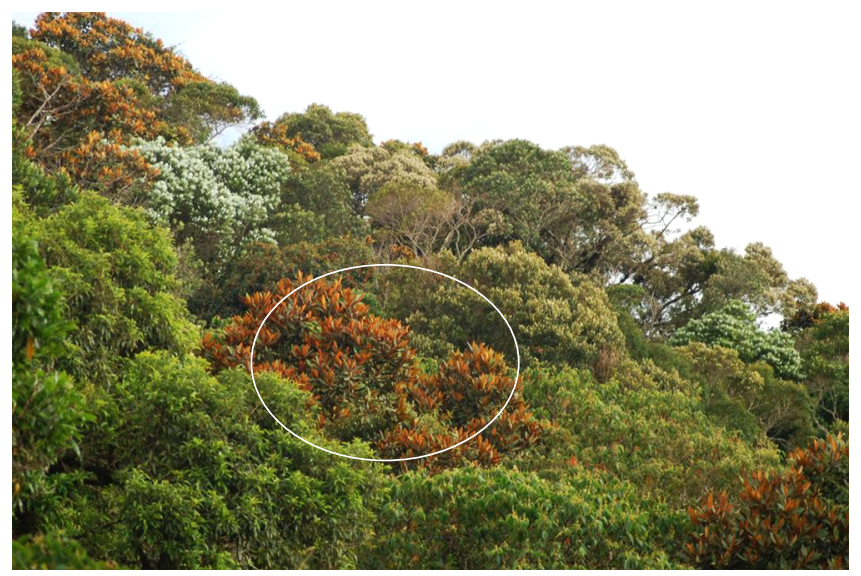

Figura 6. Aspecto da Floresta Ombrófila Densa Alto-Montana, na região do Pico do Itapeva, em Campos de Jordão, com destaque para as copas ferrugíneas de Ocotea curucutuensis. (Foto: J.B.Baitello).

Figure 6. View of the High Montane Rain Forest near the Pico do Itapeva in Campos do Jordão, with emphasis on the ferrugineous-coloured canopies of Ocotea curucutuensis. (Photograph: J.B. Baitello).
Recebido em 20/06/08

Versão reformulada recebida em 19/11/08

Publicado em 01/01/09 
